\title{
Diseño de un tesauro conceptual de folklore de la Región de Murcia
}

\author{
Miriam Tomás López \\ Universidad de Murcia (España)
}

\subsection{Resumen}

Se diseña y desarrolla un tesauro conceptual de folklore específico de la Región de Murcia denominado TEFOLKMUR que permite indizar y buscar la información pertinente en los Sistemas de Recuperación de Información. La estructura conceptual y terminológica del tesauro se ha obtenido de fuentes originales (tradición oral y protocolos notariales) y fuentes documentales impresas e inéditas. En cuanto a la metodología empleada ha sido de carácter mixto, por un lado la investigación se ha encaminado fundamentalmente hacia una metodología de corte cualitativo sustentada en una aproximación etnográfica en el área concreta de folklore y, por otro lado, hacia una investigación experimental de carácter lingüístico-cognitivo con el fin de poder analizar, interpretar, normalizar y definir los términos obtenidos de la investigación etnográfica, a través del tesauro conceptual. La estructura genérica del tesauro resultante, está formada por ocho macrodescriptores que no responden a ninguna clasificación normalizada: vestuario, instrumentos musicales, cancionero, intérpretes, comarcas de la Región de Murcia, juegos infantiles, manifestaciones de folklore y oficios populares. TEFOLKMUR forma una primera fase para que eruditos y profesionales puedan usar esta herramienta terminológica para recuperar, analizar y construir documentación relacionada con su actividad. Es un trabajo de investigación instrumental, punto de partida para otras investigaciones.

Palabras clave: Indización. Tesauro conceptual. investigación etnográfica. Folklore. Región de Murcia. Estudio de usuarios. TEFOLKMUR.

\subsection{Abstract}

A pilot conceptual specific thesaurus about the region of Murcia folklore called TEFOLKMUR is presented, that allows indexing and searching relevant information relationship with the Murcian folklore. We have obtained a thesaurus conceptual and terminology structure across the original sources (oral tradition and notarials protocols) and prints and unpublished documentary sources. We 
have used a methodology of mix character, that is, on the one hand we orient the research in the direction of a qualitative methodology based in a ethnography approximation in the folklore area and on the other hand, in the direction of experimental research of cognitive linguistic character. A structured thesaurus in eight macrodescriptors that have not a standardised classification: costumes, musicals instruments, song book, interpreters, Comarcas of Region Murcia, folklore expression, children games and popular craft. TEFOLKMUR in the first phase, so that, the erudites and professionals can used this tool for searching analysing and building documentation related with their activities.

Keywords: Indexing. Conceptual thesaurus. Ethnography research. Murcia (Region, Spain). User studies. TEFOLKMUR.

\section{Introducción}

En el trabajo que nos ocupa se ha investigado y analizado el folklore de la Región de Murcia y los resultados obtenidos de esta investigación se han canalizado y recogido en un instrumento de control terminológico, en un tesauro. El origen, diseño y construcción del mismo es propio de las Ciencias de la Documentación, pero no lo es tanto su utilización, siendo cada vez más frecuente en todas las áreas del conocimiento por ser una de las herramientas de búsqueda más exhaustivas y precisas en los Sistemas de Recuperación de Información

Se ha observado la ausencia, en la actualidad, de un lenguaje de indización en este área de conocimiento tan específica y la necesidad de converger, dentro de lo posible, en un lenguaje común, unificando la terminología aplicada a colecciones relacionadas con el folklore, tanto en las colecciones en sí como en la procedente de los informantes, es decir, los vocablos y el léxico utilizado por estas personas. Por ello, esta propuesta se basa en dos estudios que, si bien pueden parecer independientes, no se pueden desarrollar el uno sin el otro para lograr el propósito de la investigación: por un lado, el diseño y gestión de un tesauro conceptual y, por otro, el estudio de usuarios en folklore y la representación del conocimiento de los mismos a través de técnicas cualitativas.

Partiendo de la popular definición de: "Folklore es lo que sabe, siente y hace el pueblo, no lo que se sabe de él ", se deduce que es una disciplina que no se puede entender sin la presencia activa del sujeto. Es el verdadero conocedor y hacedor del patrimonio etnográfico de su zona geográfica; siente, practica y difunde el folklore, por lo que se convierte en el principal protagonista del tesauro. Se entiende por usuario aquella persona experta o no en materia de folklore que va a interrogar al sistema de información, esperando una respuesta adecuada a su nivel de conocimiento. 


\section{Delimitación conceptual}

Se enmarca el estudio en folklore, conceptualmente hablando, bajo tres aspectos fundamentales: el terminológico, el cronológico y el geográfico. Etimológicamente, el término folklore viene del inglés folk, pueblo, y lore, ciencia del saber. Es el estudio de las tradiciones, creencias y costumbres del pueblo en cada nación. En el tesauro, de estos tres elementos que encierra la definición de folklore (costumbres, ritos y creencias), se trata el aspecto artístico de cada uno de ellos, es decir, su acepción musical y todo lo que conlleva su manifestación. Cronológicamente se acota la investigación entre los siglos XV al XIX. De este último siglo procede la mayoría de las manifestaciones folklóricas que en la actualidad muestran los distintos intérpretes y grupos folklóricos. Y geográficamente se enmarca el estudio en la Comunidad Autónoma de la Región de Murcia.

Hay que indicar que el dialecto murciano no se puede enmarcar en los límites geográficos que actualmente delimitan la Región de Murcia, sino que hay que ampliar el marco geográfico e incluir sus zonas colindantes, debido a la influencia de las mismas. Según Justo Soriano (1980) el dialecto murciano limita por el Norte con el manchego, por el Este con el valenciano y por el Oeste con el andaluz, de los que ha recibido la natural influencia de vecindad.

\section{Objetivos generales}

El objetivo principal de este estudio es el diseño y desarrollo de un tesauro piloto de folklore específico de la Región de Murcia, tomando al usuario como elemento activo dentro del proceso. Independientemente de la utilidad que puede tener el tesauro para la indización y recuperación de documentos en esta materia, prima el propósito y la necesidad de lograr una normalización del lenguaje utilizado por investigadores y eruditos locales en sus trabajos folklóricos.

\section{Fuentes de Información}

Las fuentes de información utilizadas en esta investigación en su mayoría corresponden a fuentes originales y fuentes documentales impresas e inéditas

De entre las fuentes originales cabe destacar la tradición oral que, como su propio nombre indica, se transmite de forma oral, es decir, "de boca en boca". Nuestros antepasados son la fuente más pura y directa de conocimiento, de donde se puede extraer de forma veraz todo lo concerniente a las tradiciones populares. Son los guardianes de un tesoro legado por sus antepasados. El gran inconveniente que tiene la tradición oral es que, hasta que no son recogidos por un escribano, los testimonios sufren muchas variaciones al pasar de unas personas a otras; pero no sólo los acontecimientos sufren modificaciones, los vocablos (o términos) utilizados para comunicar o transmitir dichos acontecimientos, también son

Scire. $10: 1$ (en.-jun. 2004) 65-76. 
expuestos a transformaciones semánticas o sintácticas, lo que dificulta y deja en entredicho la veracidad de los datos. Sin embargo, ésta no es razón suficiente para descartar la tradición oral como fuente de información, ya que, los resultados que aporta, contrastados en todo momento con otras fuentes de información, son muy satisfactorios para el tesauro porque, a veces, constituyen la única fuente de información que existe para extraer de ella ciertos datos.

Otras de las fuentes originales revisadas han sido los protocolos notariales. De estos, se han cotejado los 390 que pertenecen a la ciudad de Jumilla, comprendidos entre los siglos XVI al XIX. La tipología documental notarial consultada en su mayoría corresponde a cartas de dote y arras, donde viene especificado el ajuar que la novia llevaba u ofrecía en su matrimonio; inventarios posmuerte, que incluyen todos los bienes que tiene el fallecido valorados por un perito para su posterior reparto entre sus más allegados; y testamentos, donde se manifiesta la declaración que hace una persona de su última voluntad, disponiendo de sus bienes y asuntos para después de su muerte. Estos protocolos proporcionan un amplio y rico vocabulario que ha sido revisado y seleccionado para el tesauro. Los términos candidatos para ser incluidos están relacionados principalmente con la vestimenta de la época.

En cuanto a las fuentes documentales impresas e inéditas consultadas cabe subrayar: los diccionarios y enciclopedias de folklore, las revistas, las monografías, las páginas Web de los distintos grupos folklóricos de la región, las fotografías antiguas y, de mención especial, el Boletín Oficial de la Región de Murcia denominado así en la actualidad y que es continuación del Boletín Oficial de la Provincia de Murcia. De este último se han revisado los años comprendidos entre 1849 y 1900. Durante estos años en los Boletines Oficiales de la Provincia se anunciaban, entre otras cuestiones, las desapariciones de personas vecinas de los pueblos de la Región. Para ello aportaban una descripción detallada del sujeto en cuestión indicando, con extraordinaria exactitud, además de sus rasgos físicos, la indumentaria que vestía en el momento de su desaparición. Para revelar realmente la precisión de sus descripciones, se muestra un ejemplo del Boletín Oficial de la Provincia de Murcia número 89 con fecha del 1 de agosto de 1849, del Juez de Primera Instancia del Partido de Cieza que cito textualmente:

[...] también se encontró un pedazo de camisa de lienzo con unas manchas de sangre en el cuello, unos pedazos de calzoncillos también de lienzo, una calceta de lana, y unos alpargates de hombre con las cintas muy largas moradas [...]

\section{Metodología mixta}

Se emplea una metodología de carácter mixto, es decir, por un lado la investigación se ha encaminado fundamentalmente hacia una metodología de corte cualitativo sustentada en una aproximación etnográfica en el área concreta del

Scire. $10: 1$ (en.-jun. 2004) 65-76. 
folklore y por otro lado; hacia una investigación experimental de carácter lingüístico-cognitivo con el fin de poder analizar, interpretar, normalizar y organizar los términos obtenidos de la investigación etnográfica.

\subsection{Investigación etnográfica}

El folklore ha carecido de bases científicas bien consolidadas y prescinde, en líneas generales, de cánones de investigación a seguir. Los investigadores folklóricos han seguido casi siempre sus propios métodos para investigar, dependiendo de sus propias necesidades de información y de las circunstancias que las rodean. Aún así, el folklore forma parte del amplio campo temático que encierra en sí misma la etnografía por lo que, etnógrafos y, concretamente, folkloristas aplican las mismas técnicas de investigación para adentrarse en esta área conceptual tan específica. Dichas técnicas, a parte de la observación participante, base fundamental de toda investigación etnográfica, son: la consulta de documentos, el análisis de contenido, entrevistas, etc.

\subsection{Técnicas cualitativas de investigación aplicadas}

Las técnicas cualitativas de investigación empleadas para la recogida de datos fueron cinco:

1. La observación participante: se adentró en la vida y realidad de los informantes, participando en sus conversaciones, en las manifestaciones folklóricas y compartiendo con ellos experiencias vividas, con el fin de obtener un amplio abanico de terminología relacionada con el folklore murciano. Los componentes o elementos fundamentales que forman parte de la observación en toda investigación etnográfica, a parte del entrevistador son: los sujetos observados y/o receptores de las entrevistas; y los escenarios o campos de observación. En cuanto a los sujetos observados el presente trabajo se llevó a cabo con personas mayores de setenta años, eruditos locales y personas que ostentan algún cargo dentro de las asociaciones regionales y grupos folklóricos; todos ellos son (o eran) vecinos de los municipios de la Región de Murcia. Otro de los elementos de la observación participante fueron los escenarios, cuya elección estuvo sujeta a una única condición: el respeto del hábitat natural de los informantes. Por ello los escenarios fueron muy heterogéneos, dependiendo del tipo de informante al que iba dirigida la entrevista: las sedes de las asociaciones regionales y grupos folklóricos, para informantes "profesionales"; hogares propios, para los eruditos locales; y jardines, Hogares de la Tercera Edad y asilos para las personas mayores.

2. La fotográfica se empleó para captar situaciones y objetos específicos relacionados con el folklore: bailes de puja, actuaciones de cuadrillas de animeros, prendas de vestuario particulares, instrumentos musicales, festivales, etc. 


\section{0}

3. Otra de las técnicas fue la entrevista individual, que se llevó a cabo tanto de forma no estructurada como semiestructurada de carácter abierto. Ambos tipos de entrevistas permitieron profundizar en las respuestas pudiendo así desarrollar otros temas que surgían en el transcurso de la entrevista y que proporcionaban información igual de valiosa y relevante que la esperada al comienzo de la misma. Desde el principio de la investigación se descartó que las entrevistas se realizaran de forma escrita por el informante, por una sencilla razón, y es que la mayoría de ellos no saben (o no sabían) leer ni escribir. Esto que, a primera vista, parece un inconveniente no era considerado como tal, ya que permitió descubrir y compartir con ellos el verdadero "saber popular".

Según Grele (1990) las entrevistas semiestructuradas son aquellas en las que el investigador prepara de antemano una lista de preguntas, pero sin limitar la entrevista a ciertas preguntas ni fijar previamente el orden en las que se contestan (May, 1997). Son flexibles y dinámicas, constituyéndose en una narración conversacional creada conjuntamente por el entrevistador y el entrevistado, que contiene un conjunto interrelacionado de estructuras que la definen como objeto de estudio. Este tipo de entrevista se ha utilizado en la investigación para recabar información de las personas mayores. De antemano se elaboraba una serie de cuestiones que servían de "guía" en la conversación mantenida con ellos (se intentaba que fuera eso: una conversación entre dos personas y no un interrogatorio donde el investigador "bombardea" a preguntas al informante), para evitar desviar demasiado del tema en cuestión. Dichas cuestiones trataban en líneas generales de su forma de vida, costumbres, fiestas, vestimenta, cantos, bailes, etc. En cuanto a las entrevistas no estructuradas no se guían por una lista preparada de preguntas, sino que se basan en el conocimiento que muestra el investigador de los propósitos de estudios (Lankshear; Knobel 2000). Dichas entrevistas fueron realizadas a aquellos informantes, que de alguna forma u otra, mantenían un vínculo de carácter profesional con el folklore en la Región de Murcia (eruditos locales), o bien, ostentaban algún tipo de cargo en una asociación o grupo folklórico (presidentes, secretarios, investigadores, etc.).

Se entrevistó a unos cuarenta y cinco informantes aproximadamente, muestra considerada razonable debido a que en las respuestas de todos ellos, salvo excepciones, llegaba un momento en que coincidían los términos referidos a un mismo concepto y sus respectivas definiciones. Es decir, cuando los diez o quince primeros informantes hablaban, por ejemplo, de la falda del traje de la mujer, todos la denominaban con alguno de estos términos: refajo, zagalejo, basquiña, saya, etc., y al seguir entrevistando a más informantes se observaba que todos repetían los mismos términos al referirse al concepto falda, y no aportaban ninguna denominación ni descripción nueva para el mismo. En definitiva el número de sujetos objeto de estudio ha sido establecido en función de lo que Juan A. Gaitán y José L.

Scire. $10: 1$ (en.-jun. 2004) 65-76. 
Piñuel (1998) denominan índice de saturación, es decir, "la muestra se considera suficiente cuando se estima una cota de información insuperable, por encima de la cual no se obtendría más información y sólo cabría esperar redundancia de información con el muestreo de más sujetos".

4. La consulta de documentos. La investigación estuvo sujeta, durante toda la fase de recogida de datos, a una continua y amplia revisión bibliográfica referida al folklore murciano.

5. Análisis de contenido y análisis del discurso. Ambas técnicas fueron aplicadas para analizar y extraer, de forma sistemática, el contenido informativo que encierran y rodean a los textos y a las conversaciones con las personas informantes. El análisis de contenido es un modo de procesar la información categorizándola en datos analizables (Bardin, 1996). Se diferencia de otras técnicas de investigación sociológica porque combina intrínsecamente la observación y producción de los datos, y la interpretación o análisis de los mismos (Andréu Abela 2001). En cuanto al análisis del discurso, Van Dijk (1985) lo define como "el estudio del uso real del lenguaje por locutores reales en situaciones reales". A lo que Stubbs (1983) añade que "el significado de una expresión depende del contexto en que se utiliza, incluyendo el contexto", es decir, no sólo hay que atenerse al sentido literal de las frases sino que también hay que deducir las posibles interpretaciones de las mismas dentro de su contexto. Ejemplos de trabajos que utilizan el análisis del discurso aplicado a la representación de estructuras conceptuales son los de María José López Huertas (1997) y Miguel Ángel López Alonso (1998), en los que el usuario queda representado en dichas estructuras de igual forma que los textos. Se pretende, con dichas técnicas, incorporar la estructura conceptual que, sobre el tema folklore, aportan los textos y los informantes en el diseño del tesauro.

\section{Diseño y elaboración de TEFOLKMUR}

Para el diseño y elaboración de este tesauro se han seguido una serie de fases o etapas establecidas, en su mayoría, en las normas UNE 50-106-90 y a las recomendaciones de Aitchison (2002). Las citadas fases son las que a continuación se detallan:

1. Identificación del tema: El tema principal del tesauro es el folklore de la Región de Murcia y los temas secundarios relacionados con él: vestuario, instrumentos musicales, cancionero, comarcas de la Región de Murcia, juegos infantiles, intérpretes, manifestaciones de folklore y oficios populares. El desarrollo de estas familias define en su totalidad el tema principal del tesauro "folklore murciano". Además de estas categorías existen otras que tratan temas marginales necesarios también para llegar a dicha definición, como sucede, por ejemplo, con la familia de los tejidos.

Scire. $10: 1$ (en.-jun. 2004) 65-76. 
2. Selección de fuentes: En la segunda fase se seleccionaron las fuentes a utilizar para extraer de ellas los términos que formarán parte del tesauro (tradición oral, protocolos notariales, manuales de folklore, diccionarios, enciclopedias, revistas, etc.).

3. Recopilación terminológica: En la que se aplicó la combinación del método deductivo e inductivo de forma simultánea. A la vez que se identificaban los términos que representaban a las categorías más amplias del tesauro, ubicando el resto de términos a dichas categorías en función de sus relaciones lógicas; en el tesauro existían categorías establecidas desde el principio de su construcción (Instrumentos Musicales y Comarcas de la Región de Murcia) lo que permitía que muchos de los términos recogidos, tras pasar por un control morfológico y semántico, se incluyeran en ellas directamente.

4. Normalización de los términos: Con el control semántico, estructural y léxico del vocabulario seleccionado se pretende llegar a una univocidad conceptual entre los términos, es decir, que existan expresiones lingüísticas con una sola

1. Cancionero

1.1. Bailes

1.2. Cancionero infantil

1.3. Cantos profanos

1.4. Cantos religiosos

1.5. Romances

2. Comarcas de la Región de Murcia

2.1. Altiplano

2.2. Campo de Cartagena

2.3. Cuenca de Mula

2.4. Noroeste

2.5. Valle del Guadalentin

2.6. Vega alta del Segura

2.7. Vega media del Segura

3. Instrumentos musicales

3.1. Cuerda

3.2. Viento

3.3. Percusión
4. Intérpretes

4.1. Campanas de Auroros

4.2. Cuadrillas de Animeros

4.3. Grupos de Coros y Danzas

4.4. Grupos profesionales

4.5. Varios intérpretes

5. Juegos Infantiles

5.1. Comienzos de juegos

5.2. Juegos con instrumentos

5.3. Juegos sin instrumentos

6. Manifestaciones de folklore

6.1. Encuentros de folklore

6.2. Festivales de folklore

7. Oficios Populares

8. Vestuario

8.1. Calzado

8.2. Labores

8.3. Tejidos

8.4. Traje Popular Femenino

8.5. Traje Popular Masculino

Tabla I. Macrodescriptores

Scire. $10: 1$ (en.-jun. 2004) 65-76. 
forma y que representen a un único concepto, desestimando las otras acepciones por no pertenecer al campo temático que nos ocupa.

5. Estructuración conceptual del tesauro: El tesauro se constituye de ocho macrodescriptores de primer nivel que no responden a ninguna clasificación normalizada. El nivel de descripción de cada uno de ellos dependerá del grado de importancia de los temas tratados dentro del contexto del folklore. Asimismo, los ocho macrodescriptores se subdividen en varios submacrodescriptores, llegando, así, al grado de especificidad requerido para comprender el árbol conceptual del tema tratado (tabla I).

6. Establecimiento de relaciones de sinonimia: Ha sido una de las fases más laboriosas en el desarrollo del tesauro debido a que el vocabulario específico de folklore murciano encierra muchos términos populares referidos a un mismo concepto. Se incluyen en el tesauro todos y cada uno de estos términos ya que el usuario final realizará las búsquedas y recuperará la información con los descriptores que él conoce. De todas las acepciones se ha intentado escoger la más representativa del concepto, bien por su frecuencia de aparición en los textos, o bien por ser la más comúnmente utilizada por el pueblo (tabla II).

Es el caso, por ejemplo de: abanico, avanillo, flabelo y ventanales. Son términos que tienen un mismo significado; abanico se toma como término preferente por ser de uso más común y significativo para los usuarios, y los términos avanillo, flabelo $y$ ventanales se toman como no preferentes, pero todos ellos tendrán entradas diferentes que reenviarán al término abanico, que es el preferente.
Abanico 8.4.1.1
UP avanillo
flabelo ventanales avanillo US Abanico flabelo US Abanico ventanales US Abanico
Tabla II. El descriptor
Abanico y sus términos equivalentes

La polisemia, al igual que la sinonimia, también ha sido muy frecuente como ocurre, por ejemplo, con el descriptor Moño de picaporte (8.4.3.3.4.1), cuyo término genérico es Moño. En materia de folklore el término "picaporte" tiene el significado de moño, por lo que se le añade el calificador de moño para precisar así su significado.

7. Establecimiento de las relaciones asociativas: Cuando las familias se organizan por agrupación de ideas en nivel horizontal se habla de relaciones asociativas. En el caso de este tesauro, las relaciones asociativas se han utilizado para vincular cada pueblo con sus cantos, con la denominación del grupo de coros y danzas y el festival que celebran. En este tesauro no son relaciones que propongan términos alternativos sino que se han establecido en función de pertenencia entre las categorías, es decir, son conceptos que no pueden pertenecer por sus significados a la misma familia pero que entre ellos tienen factores de procedencia 
que los aúnan. El resto de las familias no han sido asociadas porque sus campos conceptuales no están totalmente delimitados. Por ejemplo, en el caso de los instrumentos musicales, obligaría a relacionar todos y cada uno de los instrumentos recogidos en esta categoría con todos los pueblos de la Región de Murcia, puesto que no hay ninguno que sea propio o característico de un sólo pueblo.

8. Implementación tecnológica: El software utilizado para gestionar y soportar el tesauro fue el Beat 3.2 en versión castellana cuyo entorno permite, de forma interactiva, la construcción y consulta del tesauro.

9. Construcción del diccionario terminológico: En la última fase del diseño y desarrollo del tesauro se consideró necesario construir un diccionario terminológico como complemento adicional al tesauro de folklore, con el fin de facilitar a los usuarios la elección de los términos de búsqueda, ampliando la información contenida en el tesauro. Con este diccionario, se ha conseguido aclarar las posibles ambigüedades entre los conceptos. El Tesauro de Folklore sólo recoge cinco notas de alcance, y esto es debido a que han sido suplantadas por el diccionario terminológico, de ahí la ausencia de las mismas. Se ha considerado conveniente esta opción por la gran cantidad de términos recuperados que producen ambigüedad en su significado y que requieren de definiciones y aclaraciones más extensas que las permitidas por las notas de alcance.

7. Presentación de TEFOLKMUR. Se optó por la presentación alfabética, presentación sistemática e índice permutado por rotación. La presentación alfabética desarrolla la parte sistemática, indica qué descriptores son los más amplios y las relaciones existentes entre los mismos. Incluye, además de los términos preferentes, los no preferentes. Todos ellos ordenados de forma alfabética e indicando, en los preferentes, una notación numérica que hace referencia al lugar que ocupan en la presentación sistemática. Esta notación numérica corresponde a un código de clasificación asignado de forma correlativa en la presentación sistemática. La presentación sistemática muestran las relaciones jerárquicas entre los conceptos. El índice permutado por rotación se compone de todos los términos del tesauro, tanto los descriptores como los no descriptores, ordenados alfabéticamente por todas las palabras significativas que contienen.

\section{Contenido general del tesauro}

A modo de resumen, el tesauro lo constituyen mil quinientos cuarenta y nueve términos en total, de los cuales mil trescientos setenta y cinco son preferentes y ciento setenta y cuatro son términos no preferentes. Existen ocho términos superiores y mil doscientos cinco inferiores. Estas cifras demuestran que es un tesauro con una gran riqueza terminológica ya que, eligiendo ocho categorías exclusivamente, y siendo el tema abordado susceptible de incluir muchas más, 
se ha logrado rescatar, clasificar, estructurar y definir una gran cantidad de terminología folklórica. En cuanto a las relaciones existentes hay ciento setenta y seis relaciones de equivalencia, mil trescientas cincuenta y cinco jerárquicas y cuatrocientas treinta y dos asociativas.

\section{Conclusiones}

El paso de la oralidad a la escritura, recopilando y estructurando la terminología relacionada con el folklore, permite salvar una rica tradición popular que, de otro modo, puede sucumbir en el tiempo y el olvido. El intento de normalizar la terminología relacionada con el folklore de la Región de Murcia posibilitará a los investigadores y eruditos locales utilizar con propiedad los términos y conceptos en sus trabajos de investigación. También ayudará a unificar distintos significados referidos a un mismo término que, hasta el momento, eran empleados indistintamente según el autor o informante.

En el Tesauro Conceptual de Folklore de la Región de Murcia, la presencia del usuario ha sido de vital importancia y pieza fundamental y decisiva para la construcción del mismo. La aplicación de las técnicas de investigación cualitativa en Biblioteconomía y Documentación no sólo ayuda a estudiar el comportamiento de los usuarios en la fase de búsqueda y recuperación de información; sino que, además permite extraer de ellos los diferentes puntos de vista y las estructuras conceptuales del campo de estudio en cuestión.

El Tesauro Conceptual de Folklore de la Región de Murcia será, con su aplicación, una herramienta de gran utilidad y fácil manejo para los usuarios en sus búsquedas y recuperación de información, porque ha sido diseñado y desarrollado dentro del contexto para el que ha sido creado. Forma un primer peldaño para que eruditos y profesionales puedan usar esta herramienta terminológica para recuperar, analizar y construir documentación relacionada con su actividad, y un punto de partida para sucesivas investigaciones.

\section{Referencias}

Aitchison, J. (2000). Thesaurus construction and use: a practical manual. 4nd. ed. London: Aslib, 2000.

Andréu Abela, J. (2001). Las técnicas de Análisis de Contenido: una revisión actualizada Granada. Fundación Centro de Estudjos Andaluces, 2001. URL: <http: //www.fundacion-centra.org/pdfs/S200103.PDF >. Consultado: 2002-08-20.

Asensi, V. ; Pastor, J. A. Propuestas de un modelo de interfaz genérica para sistemas de recuperación de información. // SCIRE. Representación y Organización del Conocimiento. 4:1 (en.-jun. 1998) 71-88.

Asociación Española de Normalización. Directrices para el establecimiento de tesauros monolingües. Norma española. UNE 50-106-90. Madrid: AENOR, 1994.

Scire. $10: 1$ (en.-jun. 2004) 65-76. 


\section{Miriam Tomás López.}

Bardin, L. (1996). Análisis de contenido. 2nd. ed. Madrid: Akal, 1996.

Dijk, T.A Van (1985). Handbook of Discourse Analysis, Volume 4: Discourse Analysis in Society. London: Academic Press, 1985.

Gaitán Moya, J.A. ; Piñuel Raigada, J.L. (1998). Técnicas de investigación en comunicación social: elaboración y registro de datos. Madrid: Síntesis, 1998.

García Soriano, J. (1980). Vocabulario del dialecto murciano. Murcia: Editora Regional, 1980.

Grele, R.J. (1990). La historia y sus lenguajes en la entrevista de historia oral: quién contesta a las preguntas de quién y porqué. // Historia y Fuente Oral. 5 (1990) 111-129.

Ingwersen, P. (1996). Cognitive perspectives of information retrieval interaction: Elements of a cognitive IR theory. // Journal of Documentation. 52:1 (1996) 3-50.

Lancaster, F.W.; Pinto, M. (Coords.) (2001). Procesamiento de la información científica. Madrid: Arco/Libros, 2001.

Lankshear, C. ; Knobel, M. (2000). Problemas asociados con la metodología de la investigación cualitativa. // Perfiles Educativos. 87 (2000) 6-27. URL: <http: //www.cesu.unam.mx/iresie/revistas/perfiles/perfiles/87-html/87-02.htm>. Consultado: 2002-07-03.

López Alonso, M.A. (1998). Un tesauro conceptual para la recuperación de la información jurídica comercial. // Revista Española de Documentación Científica. 21:2 (Abr. -jun. 1998) 164-173.

López-Huertas Pérez, M.J. (1997). Thesaurus structure design: A conceptual approach for improved interaction. // Journal of Documentation. 53:2 ( March 1997) 139-177.

May, T. (1997). Social research: issues, methods and process. 2nd. ed. Buckingham: Open University Press, 1997.

Stubbs, M. (1983). Análisis del discurso: análisis sociolingüístico del lenguaje natural. Madrid: Colección Alianza Psicológica, 1983.

Scire. $10: 1$ (en.-jun. 2004) 65-76. 